\title{
CONTRIBUTION DES ASTROLABES A LA DETERMINATION DU SYSTEME DE REFERENCE DANS L'HEMISPHERE SUD
}

\author{
G. BILLAUD and S. DEBARBAT \\ Observatoire de Paris, France
}

\begin{abstract}
The potentialities of astrolabe observations which are available for improving the fundamental system, particularly in the southern hemisphere, are discussed. Difficulties arise because of the poor distribution in latitude of the observing stations and diversity of observational programmes.
\end{abstract}

\section{Généralités}

L'observation répétée des mêmes étoiles met en évidence des erreurs systématiques dues, pour une large part, aux erreurs du catalogue fondamental et leur correction est l'un des buts que poursuivent les astronomes des deux hémisphères. Ils disposent pour cela de divers instruments; parmi ceux-ci, bien qu'il ne puisse atteindre les étoiles au-delà de la magnitude 6,0, l'astrolabe permet l'étude de $91 \%$ des étoiles du FK4 et de 55\% de celles du FK4 Supp, avec une précision (écart-type d'une mesure en distance zénithale) de l'ordre de 0 ". 17 en moyenne. A ce titre, il est susceptible d'apporter une contribution importante à l'amélioration du catalogue fondamental.

Les conditions d'élaboration d'un catalogue astrolabe sont bien connues(Débarbat et Guinot, 1970). De tels catalogues sont en principe dépourvus d'équations de couleur et de magnitude, ainsi que d'erreurs du type $\Delta \alpha_{\alpha}, \Delta \alpha_{\delta}, \Delta \delta_{\alpha}$. Il subsiste toutefois :

(a) Une erreur constante des ascensions droites.

(b) Une erreur systématique des déclinaisons du type $\Delta \delta_{\delta}$ de forme connue, d'amplitude faible mais indéterminée.

Dans ces catalogues la précision en ascension droite est proportionnelle à $1 / \cos \varphi \sin a$ et celle en déclinaison à $1 / \cos S(\varphi, a, S$ désignent respectivement latitude, azimut et angle parallactique). Ceci entraîne que pour une latitude donnée, il existe, en ascension droite, deux zones de même précision, l'une vers le pôle, l'autre vers l'équateur, condition que l'on ne trouve pas dans les catalogues établis avec un instrument méridien où la précision dépend de $\cos \delta$. La même chose existe en déclinaison, où il faut remarquer la présence d'une zone d'indétermination qui couvre de 10 à 15 degrés en $\delta$.

\section{Résultats obtenus}

Les catalogues réalisés ont montré que la méthode conduisait à des résultats sûrs et les craintes d'introduction d'erreurs supplémentaires dues à des anomalies de réfraction se sont montrées non fondées. Cependant, les résultats actuels ne justifient pas l'espoir que l'on avait eu lors de la première publication sur ce sujet (Guinot, 1958), malgré la très forte concentration d'astrolabes dans l'hémisphère nord, les résultats 
sont loin de correspondre aux possibilités réelles. Selon toute vraisemblance, cela tient au fait que l'astrolabe devait fournir en premier lieu heure et latitude et que les corrections aux positions du FK4 n'apparaissaient que comme un sous produit du programme fondamental, lui-même inadapté à l'établissement d'un catalogue: lorsqu'un programme spécial est absent, le nombre d'étoiles observées est insuffisant pour une étude systématique; de plus, cette insuffisance est fréquemment aggravée par le manque d'homogénéité des programmes d'observation. Les zones de recouvrement existent, mais il est exceptionnel qu'elles contiennent plus de 50 étoiles, ce qui est très peu.

Le tableau ci-dessous donne les valeurs des coefficients de recouvrement, c'est-àdire le nombre d'étoiles communes à deux catalogues par degré de déclinaison; les valeurs inscrites sur l'hypothénuse du triangle fournissent la densité du catalogue.

Les observatoires mentionnés couvrent de $-60^{\circ}$ à $+77^{\circ}$ en déclinaison.

TABLEAU I

\begin{tabular}{lllllllllll}
\hline & Pt & H & P & N & W & A & C & Q & T & S.J. \\
\hline Potsdam & 2,1 & & & & & & & & & \\
Herstmonceux & 0,9 & 2,5 & & & & & & & & \\
Paris & 1,8 & 2,1 & 6,0 & & & & & & & \\
Neuchatel & 0,7 & 0,8 & 1,6 & 2,3 & & & & & & \\
Washington & 0,4 & 0,5 & 1,1 & 0,4 & 1,5 & & & & & \\
Alger & 0,2 & 0,3 & 1,0 & 0,1 & 0,3 & 1,7 & & & & \\
Curaçao & 0,4 & 0,4 & 1,0 & 0,2 & 0,0 & 0,2 & 1,3 & & & \\
Quito & 1,0 & 1,9 & 2,5 & 0,9 & 0,4 & 0,2 & 0,3 & 2,9 & & \\
Tananarive & & & & & & 0,7 & 0,2 & 0,6 & 1,9 & \\
San Juan & & & & & & & 0,6 & 0,4 & 0,1 & 1,8 \\
\hline
\end{tabular}

Dans l'hémisphère sud on constate les mêmes difficultés que dans l'hémisphère nord, accrues par une couverture encore plus faible. A cet égard on ne peut que regretter l'interruption des observations au Cap $\left(-33^{\circ} .9\right)$ et à Wellington $\left(-41^{\circ} 3\right)$.

\section{Projets pour l'hémisphère sud}

L'exploitation des résultats des deux stations temporaires de Curaçao $\left(+12^{\circ}\right)$ et de Tananarive $\left(-19^{\circ}\right)$ (Année Géophysique Internationale) a permis la publication de deux petits catalogues de 77 et 113 étoiles respectivement. L'observatoire de Quito $\left(0^{\circ}\right)$ a, de son côté, publié un catalogue de 202 étoiles: il est inutile d'insister sur son importance dans le raccordement des deux hémisphères.

A ces publications, devraient se joindre bientôt le catalogue de Santiago du Chili $\left(-33^{\circ}\right)$, de San Juan $\left(-32^{\circ}\right)$, le second catalogue de l'observatoire de Quito ainsi que le catalogue de l'observatoire du Cap et peut-être celui de Wellington.

Ces perspectives encourageantes montrent tout l'intérêt que les astronomes de l'hémisphère sud portent à ces travaux. Il convient de noter à ce sujet une entreprise 
originale des astronomes d'Amérique Latine qui ont suscité en novembre 1971, à l'occasion du centenaire de l'observatoire de Cordoba (République Argentine), le 'First Latin American Astrometric Working Meeting'. Au cours de cette réunion, où l'on s'est tout particulièrement penché sur les problèmes d'homogénéisation et de coordination, plusieurs recommandations ont été approuvées.

Outre les prochaines publications de catalogues que nous avons déjà signalées s'inscrivent, dans les projets à court terme, la mise sur pied de stations dans l'extrême sud américain $\left(-53^{\circ}\right.$ et $\left.-46^{\circ}\right)$. Ces projets doivent être fortement encouragés, car si les conditions climatiques le permettent, l'élaboration d'un catalogue, couvrant en déclinaison une zone allant de $-20^{\circ}$ à $-80^{\circ}$, apporterait à la connaissance du ciel austral une contribution essentielle.

L'importance des erreurs du catalogue fondamental pour les déclinaisons négatives justifie la publication de catalogues provisoires établis à partir d'un moins grand nombre d'observations que dans l'hémisphère nord; le problème est en effet analogue à celui qu'a traité le Professeur Lacroute lors du Colloque no. 20 à Copenhague dans un domaine voisin. Concrètement il apparait qu'avec un an d'observations de qualité moyenne, correspondant à un écart quadratique moyen de 0 ". 25 , on disposerait d'un catalogue préliminaire intéressant, mettant en évidence les erreurs du FK4 d'une manière significative.

\section{Projet d'un catalogue général astrolabe}

Il reste ensuite à raccorder entre eux les différents catalogues des deux hémisphères. Les études faites à Paris ont montré la possibilité, à partir des corrections des étoiles communes, de réaliser un catalogue homogène. Ce catalogue est en cours d'exécution; il est pratiquement achevé en ce qui concerne les $\alpha$. Il comprendra en particulier un fichier où seront répertoriées les contributions des divers observatoires, leurs écarts systématiques ainsi que le jeu des corrections d'harmonisation de chacun d'eux. La révision lors de l'adjonction d'un nouveau catalogue ne présentera aucune difficulté.

La qualité du raccordement sera grandement améliorée si une nouvelle station équatoriale peut être créée. Cette création aurait des conséquences importantes non seulement pour les catalogues mais aussi pour la détermination du temps et de la latitude.

D'autres possibilités sont ouvertes. Krejnin (1968) a en effet montré qu'il était possible d'obtenir, en associant deux astrolabes travaillant à des distances zénithales différentes, un catalogue de déclinaisons absolues. Un tel programme de recherche pourrait être poursuivi dans les prochaines années en utilisant l'astrolabe à pleines pupilles (Billaud et Guinot, 1971), dont il existe à l'Observatoire de Paris un prototype en service permanent. Deux autres instruments actuellement en cours d'étude seront aussi adaptés à l'emploi de la méthode de Krejnin: il s'agit du Reflecting Astrolabe (Herstmonceux) et de l'Astrolabe Photoélectrique (CERGA, Grasse).

Comme on l'a vu, les observations d'étoiles à l'astrolabe ne permettent pas de fixer l'équinoxe: cela ne peut être fait directement qu'en observant le Soleil et une telle 
entreprise présente de grandes difficultés. Il existe cependant une méthode indirecte de rattachement : c'est l'observation des planètes. Aux observatoires de l'hémisphère nord: Alger, Besançon, Paris, San-Fernando se sont déjà joints les observatoires de Quito et de Sao Paulo, auxquels se joindront bientôt ceux de San Juan et de Santiago du Chili, permettant l'observation de Mars, Jupiter, Saturne, Uranus et Vesta ainsi qu'il a été recommandé par le colloque UAI no. 20 (Copenhague 1972). Une collaboration internationale est nécessaire pour assurer la couverture des trajectoires avec le maximum de précision, celle-ci dépendant, selon la déclinaison, de la latitude du lieu d'observation.

\section{Conclusion}

Ainsi l'astrolabe apparaît comme un outil efficace pour l'amélioration du système de référence, tout particulièrement dans l'hémisphère sud. La tâche entreprise doit être poursuivie, et menée avec le souci de satisfaire aux nécessités des raccordements entre catalogues.

\section{Bibliographie}

Billaud, G. et Guinot, B.: 1971, Astron. Astrophys. 11, 241.

Débarbat, S. et Guinot, B.: 1970, La méthode des hauteurs égales en astronomie, Gordon and Breach.

Guinot, B.: 1958, Bull. Astron. 22, 1.

Krejnin, E. I.: 1968, Astron. Zh. 45, 447.

\section{DISCUSSION}

Fricke: There is no doubt about the importance of the contributions obtained with astrolabes for the improvement of the fundamental system. Miss Débarbat's request for a more rational world-wide organisation of such observations is justified. I am sure that IAU Commission 8 will be willing to adopt a resolution to this effect. 